\title{
Synthesis and Characterization of Cross-linked Polysiloxane Microspheres for Light-scattering
}

\author{
Peng-wei Yin ${ }^{1,2, a}$, Ming-shan Yang ${ }^{1,2, b^{*}}$, Hong-liang Tong ${ }^{1,2, c}$
}

1. Department of Material Science and Engineering, Beijing Key Lab of Special Elastomer Composites Materials, Beijing Institute of Petrochemical Technology, Beijing 102617, China

2. College of Materials Science and Engineering, Beijing University of Chemical Technology,Beijing,100029,China

a.pengweiyin@126.com, b. yangms001@126.com, c.735824830@qq.com

${ }^{*}$ Corresponding author

Keywords: Polysiloxane , Microspheres, Light Scattering

\begin{abstract}
The narrow particle size distribution of poly (vinyl silicone) microspheres were prepared by the hydrolysis-polycondensation method with vinyl silane (VTMS) as raw materials. And the influences of $\mathrm{pH}$ value, oil/water ratio on the morphology, particle size and distribution of the microspheres were studied. The results shows that the particle size of microspheres decrease with the increasing of the $\mathrm{PH}$ value of polymerization, and the particle size has no specific linear relationship with the $\mathrm{PH}$ value of hydrolysis reaction, while the oil-water ratio is smaller, single particle size distribution is more narrow, more uniform distribution.
\end{abstract}

\section{Introduction}

At present, LED energy-saving lamps are widely used in various lighting fields due to their low energy consumption, high brightness, long life and other advantages, including a variety of indoor lighting, TV products. But the light emitted by the LED lamp is strong and harsh, so that people can not look directly, can not achieve the soft visual effect, and the light diffusion products will be a good solution to these problems [1][2]. The light diffusing agent is applied in various lamp shades, which can make the point light source to the surface light source, and the light is soft and the brightness is high. Formerly the light diffusion agent use inorganic particles, such as silicon dioxide, although the fog of LED lights increased, the transmittance also decreased, affecting the efficiency of LED[2]. So the organic light diffusing agent has been developed, which mainly include PMMA and organic silicon microsphere. Polysiloxane microspheres' (PSQ) chemical formula is RSiO3/2, side group $\mathrm{R}$ is an organic group connected with silicon atoms, such as methyl, vinyl, phenyl, amino, mercapto, epoxy [3]. PSQ includes both inorganic silicone "skeleton" structure and also the ball's shape side groups of organic groups in the molecular structure. This special chemical composition and morphology make it have the excellent properties of inorganic/organic hybrid materials and the unique effect of micron size particles, which can be used as a light diffusing agent for LED lamp shade [4]. At present, the domestic market of organic light diffusion microspheres are virtually monopolized by foreign companies such as supply, Shin-Etsu, they're expensive. Therefore, the independent research and development of a narrow particle size distribution, high transmittance and high haze of light diffusion materials has a very important significance for further implementation of LED lighting and energy-saving emission reduction[5]. In this paper, we make Poly vinyl silicone microsphere by the two step method of hydrolysis and condensation polymerization, and this method using VTMS as raw material. The influences of microspheres size and distribution by concentration of ammonia, oil water ratio and reaction temperature were studied. 


\section{Experiments}

Material. VTMS(Vinyl trimethoxy methyl silane), AR, supplied by Tianjin Fuchen Chemical reagents factory; Polyvinylpyrrolidone(PVP), AR, supplied by Aladdin; hydrochloride, AR, supplied by Beijing Chemical Reagents Company; ammonia, AR, supplied by Beijing Chemical Reagents Company; Absolute alcohol, AR, supplied by Beijing Chemical Reagents Company; Deionized water, prepared in the lab.

Preparation. Added a certain amount of VTMS,PVP and hydrochloride to the three mouth flask of $250 \mathrm{ml}$, equipped with a thermometer and blender, then put the flask in a water bath and controlled the temperature. Slowly adding hydrochloric acid to control its $\mathrm{pH}$ value. Start a hydrolysis reaction, adding proper amount of ammonia to three flasks, adjusting the $\mathrm{pH}$ value. The samples which obtained by Hydrolysis and polymerization were centrifugated(or suction filtration)and precipitated, then washed 2times by absolute alcohol, and then washed them 1 time with deionized water to remove the samples without the reactant and the residual monomer, finally vacuum drying at $100^{\circ} \mathrm{C}$ for 10 hours.

Characterization. Granular Analysis: Measured particle size $(\mu \mathrm{m})$ and span size $(\%)$ of microspheres by laser particle size distribution analyzer manufactured by Dandong Bettersize Instruments Co., Ltd., China. The morphology of the microspheres were observed by SEM using the working voltage of $0 \sim 200 \mathrm{kV}$.

\section{Results and Discussion}

Effect of oil water ratio on particle size and distribution. In the experimental process, keep the $\mathrm{pH}$ value of hydrolysis was 4 , the reaction temperature was $25^{\circ} \mathrm{C}$, the hydrolysis time was $4.0 \mathrm{~h}$, the polymerization $\mathrm{pH}$ value was 8.5 , and the polymerization time was $2.0 \mathrm{~h}$, the results of effect of oil water ratio on particle size and distribution was shown in Table 1.

Table 1 Effect of different oil / water ratio on the particle size of microspheres

\begin{tabular}{c|c|c|c|l|l|l}
\hline Number & $\mathrm{n}_{\text {siloxane }} / \mathrm{n}_{\text {water }}$ & $\begin{array}{c}\text { hydrolysis } \\
\mathrm{pH} \text { value }\end{array}$ & $\begin{array}{c}\text { polymerization } \\
\mathrm{pH} \text { value }\end{array}$ & Product form & $\begin{array}{l}\text { Particle } \\
\text { size/ } \mu \mathrm{m}\end{array}$ & Distribution \\
\hline $3.24-1$ & $1: 7$ & 4.0 & 8.5 & Microspheres & 12.680 & 4.539 \\
\hline $3.24-2$ & $1: 17$ & 4.0 & 8.5 & Microspheres & 9.639 & 4.568 \\
\hline 3.17 & $1: 17$ & 4.0 & 8.0 & Microspheres & 14.44 & 4.358 \\
\hline 3.23 & $1: 17$ & 3.0 & 8.5 & Microspheres & 23.14 & 3.101 \\
\hline
\end{tabular}

As can be seen from the above, with the smaller the oil/water ratio, the particle size distribution was more narrow, and the distribution was more uniform.

Effect of polymerization $\mathrm{pH}$ value on the particle size and distribution of microspheres. The results of effect of polymerization $\mathrm{pH}$ value on the particle size and distribution of microspheres were shown in Table 2.

Seen from Table 2, with the increase of $\mathrm{OH}$ - concentration in the polymerization reaction, the particle size decreased gradually. This is because the concentration of $\mathrm{OH}$ - ion in the solution is lower, the condensation reaction is slow, and the product has a lower degree of cross-linking, which is part of the oligomer. Because ammonia as the polycondensation catalyst, with the concentration increased, the concentration of $\mathrm{OH}_{-}$in solution increased, alkali catalytic effect obviously, the reaction rate increased, in a very short period of time that generate a large number of particles precipitating, resulting in the formation of polyvinylsiloxane particles with smaller particle size. 
From Table 1 can see $\mathrm{pH}$ value of polymerization is not a linear influence on particle size distribution.

Table 2 Effect of polymerization $\mathrm{pH}$ value on the particle size of microspheres

\begin{tabular}{c|c|l|c|c}
\hline Number & $\mathrm{pH}$ value & Product form & Particle size $/ \mu \mathrm{m}$ & Distribution \\
\hline 4.13 & 7.0 & Microspheres & $\bullet 4.468$ & 4.418 \\
\hline 4.19 & 8.0 & Microspheres & 2.832 & 13.430 \\
\hline 3.31 & 9.0 & Microspheres & 2.416 & 9.634 \\
\hline 4.07 & 10.0 & Microspheres & 2.303 & 9.095 \\
\hline 3.30 & 10.5 & Microspheres & 0.909 & 11.040 \\
\hline 4.06 & 11.0 & Microspheres & 0.643 & 0.643 \\
\hline
\end{tabular}

Effect of pH value on the morphology of microspheres. The microspheres were scanned by electron microscope such as Figure 1 and Figure 2.

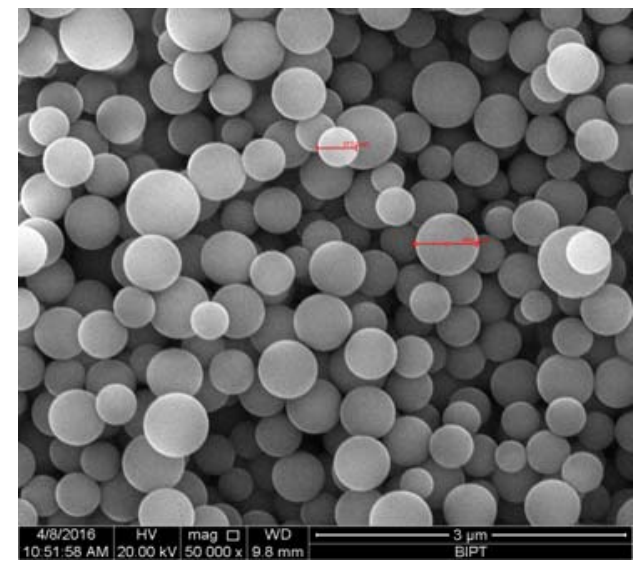

Figure 1 Scanning electron microscope

photographs of the number 3.31

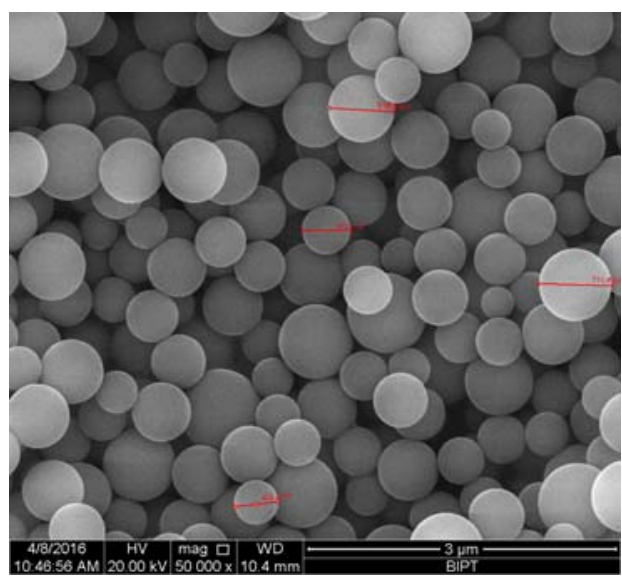

Figure 2 Scanning electron microscope

photographs of the number 4.07

It can be seen from the Figure 5 and 6, polysiloxane microspheres prepared by this experiment was indeed spherical appearance, But sample number 4.07 particle size distribution was more uniform, and the spherical degree was better. It was also consistent with the results measured by the laser particle size distribution analyzer. If there was hydrogen bonding, the microspheres are easy to reunite.

\section{Conclusion}

The narrow particle size distribution of poly (vinyl silicone) microspheres were prepared by the hydrolysis-polycondensation method with vinyl silane (VTMS) as raw materials. The results showed that the particle size of microspheres decrease with the increasing of the $\mathrm{PH}$ value of polymerization, while the oil-water ratio is smaller, single particle size distribution is more narrow, more uniform distribution. 


\section{Acknowledgement}

This research was financially supported by the Graduate Innovation Foundation(16033981001/030) of Beijing Institute of Petrochemical Technology, China.

\section{References}

[1] X.T. Yan, J.F. Yang, B. Xue, et al. Design of a novel LED collimating element based on freeform surface[J]. Optoelectronics Letters(Chinese), 9 (2013): 9 12.

[2] G.Y. Yu, X.P. Zhu, S.H. Hu, et al. Thermal simulation and optimization design on a high-power LED spot lamp[J]. Optoelectronics Letters(Chinese), 7(2011): 117 121.

[3] C. R. Miller, R. Vogel, P. P. T. Surawski, et al. Functionalized organosilica microspheres via a novel emulsion-based route[J]. Langmuir, 21(2005) : 9733-9740.

[4] D.L. Qi, X.L.Yang, W.Q. Huang. Influence of cross-linking degree on the formation of monodisperse polymer microspheres in the process of distillation precipitation polymerization [J]. Ion exchange and adsorption(China), 21(2005) : 481- 486.

[5] Q.H. Meng, A.R. Guo, Y.J. Zhang. Lighting scattering property of PS/PMMA compounds[J]. Optics and Precision Engineering(China), 17(2009): 646-650. 